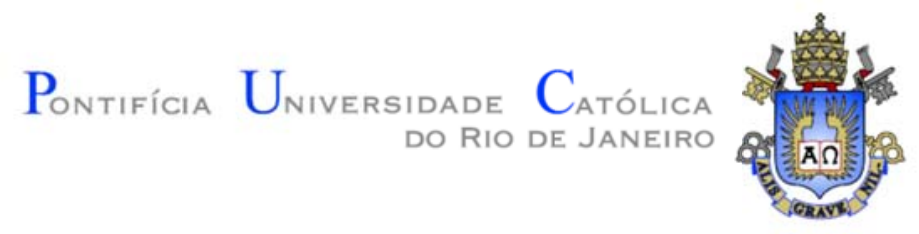

Pablo Frias de Oliveira Bioni Gomes

\title{
Extração de Primeiro Plano em Imagem HD com Fundos Variados
}

Dissertação apresentada ao Programa de Pós-graduação em Informática da PUC-Rio como requisito parcial para obtenção do título de Mestre em Informática.

Orientador: Prof. Bruno Feijó 


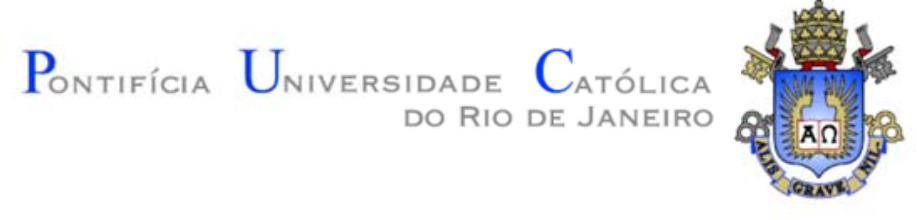

Pablo Frias de Oliveira Bioni Gomes

\title{
Extração de Primeiro Plano em Imagens HD com Fundos Variáveis
}

\begin{abstract}
Dissertação apresentada como requisito parcial para obtenção do grau de Mestre pelo Programa de Pósgraduação em Informática da PUC-Rio. Aprovada pela Comissão Examinadora abaixo assinada.
\end{abstract}

Prof. Bruno Feijó

Orientador

Departamento de Informática - PUC-Rio

Prof. Raul Queiroz

Departamento de Eng. Elétrica - PUC-Rio

Prof. Luiz Eduardo A. Sauerbronn

Departamento de Eng. Mecânica - UFRJ

Prof. José Eugênio Leal

Coordenador Setorial do Centro

Técnico Científico - PUC-Rio

Rio de Janeiro, 16 de março de 2010 
Todos os direitos reservados. É proibida a reprodução total ou parcial do trabalho sem autorização da universidade, do autor e do orientador.

\section{Pablo Frias de Oliveira Bioni Gomes}

Graduou-se em Engenharia de Computação na Pontifícia Universidade Católica do Rio de Janeiro em 2004.

Ficha Catalográfica

Gomes, Pablo Frias de Oliveira Bioni

Extração de Primeiro Plano em Imagem HD com Fundos variados / Pablo Frias de Oliveira Bioni Gomes; orientador: Bruno Feijó. - Rio de Janeiro : PUC-Rio, Departamento de Informática, 2010.

v., 55 f: il. ; $30 \mathrm{~cm}$

1. Dissertação (mestrado) - Pontifícia Universidade Católica do Rio de Janeiro, Departamento de Informática

Incluí referências bibliográficas.

1. Informática - Teses. 2. Extração de Fundo 3. Processamento digital de Imagem. I. Feijó Bruno. II. Pontifícia Universidade Católica do Rio de Janeiro. Departamento de Informática. III. Título. 


\section{Agradecimentos}

Ao meu Avó pór ter me dado meu primeiro computador.

A minha família pelo suporte em todos os momentos difíceis desta caminhada.

Aos grandes amigos que me acompanharam por este período, Carlos Andre Tavares Campos, Rodrigo Marques, Aarão Irving, Francisco Lima, Marcelo Nicácio.

A minha amiga especial de todas as horas e momentos Elizabeth Ferraz, pelo grande apoio e motivação.

Ao meu orientador Bruno Feijó e família, pela grande dedicação ao longo do processo todo, de forma incansável. 


\section{Resumo}

Gomes, Pablo Frias de Oliveira Bioni; Feijó, Bruno. Extração de Primeiro Plano em Imagem HD com Fundos Variados. Rio de Janeiro, 2010. 55p. Dissertação de Mestrado - Departamento de Informática, Pontifícia Universidade Católica do Rio de Janeiro.

A indústria de cinema e TV tem usado amplamente a técnica de Chroma Key, também conhecida por Blue Screen Matting. Esta técnica revolucionou, ao longo do tempo, a indústria do entretenimento, permitindo que cenas impossíveis de serem criadas se tornassem realidade. A evolução dessa técnica permitiu que produções complexas passassem a ter melhor controle e custos mais baixos. Porém, essa técnica conta com uma série de etapas de preparação, que demandam recursos financeiros elevados e planejamento preciso. Ademais, erros de continuidade costumam criar sérios problemas na pós-produção.

Atualmente, a indústria de entretenimento está procurando outras técnicas de matting que funcionem com fundos variados. O uso destas técnicas ainda está restrita a trabalhos acadêmicos e a softwares de manipulação de imagens estáticas.

O presente trabalho tem como objetivos fazer uma análise dos processos atuais de chroma key e partir para a proposta de uma técnica de matting com fundos variados em imagens de alta definição (HD - High Definition). Dois métodos para o cálculo de valores de alpha são apresentados: um método global baseado em clusters e um método local baseado em potencial elétrico.

\section{Palavras-chave}

Chroma key; Matting de imagem; Efeitos Especiais; Composição 


\section{Abstract}

Gomes, Pablo Frias de Oliveira Bioni; Feijó, Bruno (Advisor). Foreground

Extraction in HD Images on Complex Backgrounds . Rio de Janeiro, 2010. 55p. MSc. Thesis - Departamento de Informática, Pontifícia Universidade Católica do Rio de Janeiro.

The film and broadcast industry have been massively using the Chroma Key technique, also known as Blue Screen Matting. This technique deeply transformed the entertainment industry, allowing impossible scenes become reality. The evolution of this technique allowed that complex productions could have better control and lower costs. However, this technique needs a sequence of preparation stages, which require high budgets and precise planning. Furthermore, continuity errors usually cause serious post-production problems.

Currently, the entertainment industry is searching for other matting techniques that work on any kind of background. The use of these techniques is still restricted to academic works and softwares of still image manipulation.

The present work has the goal of making an analysis of the current chroma key processes and aims to propose a matting technique over any type of background in High Definition images. Two methods of calculating alpha values are presented: a local method based on clusters and a local one based on electric potential.

\section{Keywords}

Chroma key; Image matting; Special Effects; Compositing 


\section{Sumário}

1 Introdução 15

1.1. O Processo Atual de Composição na Indústria 15

1.2. Chromaless 17

1.3. Objetivos e Características 18

1.4. Tradução de Termos

1.5. Organização da Dissertação 21

2 Matting e Chroma Key 22

2.1. O Problema de Matting 22

2.2. Categorias de Algoritmos de Matting 26

2.3. Chroma Key 28

3 Trabalhos Relacionados 34

3.1. Sistemas de Chroma Key de Mercado 34

3.1.1. Primatte Keyer 34

3.1.2. Keylight Keyer 36

3.1.3. IBK Keyer 38

3.1.4. Chromatte 39

3.2. Trabalhos de Pesquisa em Matting 40

4 Uma Proposta de Técnica Chromaless 42

4.1. Círculo de Amostragem 42

4.2. Cálculo de Alpha 43

4.3. Tratamento Global com Clusterização por K-Means 45

5 Resultados, Conclusões e Trabalhos Futuros 47

5.1. Resultados e Conclusões 47

5.2. Trabalhos Futuros 51 


\section{Lista de Figuras}

Figura 1.1 Exemplo da técnica de chroma key com fundo azul [imagem extraída de Long Road (2007), referente ao filme 300]

Figura 1.2 Backlot para cena do filme Guerra dos Mundos (War of the Worlds) [Imagem sob GNU FD License, Wikimedia Commons, 23 May 2007].

Figura 1.3 Digital Backlot com reconstrução parcial do ambiente [cortesia de Francisco Lima, VFX Supervisor, TV Globo, 2009]

Figura 2.1 Imagem I como uma combinação convexa da imagem foreground $\mathrm{F}$ e a imagem background $\mathrm{B}$ usando o coeficiente de interpolação $\alpha$. O coeficiente $\alpha$ é chamado de "matte". [imagem extraída de Wang (2007)].

Figura 2.2 Exemplo de defeitos de composição após a extração do foreground. A composição sem defeitos aparentes é obtida com o algoritmo de Wang \& Cohen (2007a). [Figura composta a partir de Wang \& Cohen 2007a]

Figura 2.3 O sistema Drag-and-Drop Pasting (Jia et al. 2006), onde a região selecionada pelo usuário é colocada na imagem alvo (b), a região é otimizada (c) e a composição final é obtida (d) [Figura composta a partir de Jia et al. 2006].

Figura 2.4 Exemplo de trimap (b) e matte obtido a partir deste trimap (c) [Extraído de Wang \& Cohen (2008)].

Figura 2.5 No algoritmo Primatte, $\alpha$ é dado pela distância do pixel C para o poliedro B no espaço de cor RGB [figura adaptada de Wang \& Cohen (2008)]. 
Figura 2.7 Fluxograma do processo de chroma key digital

Figura 2.8 Ruídos nos canais

Figura 2.9 Padrão de Bayer para disposição de sensores usado pela maioria das câmeras digitais [Imagens sob GNU FD License, Wikimedia Commons, 28 Dezembro 2006]

Figura 3.1 Caixas Inner (background) e Outer no espaço RGB para algoritmos de chroma key

Figura 3.2 Gradação tonal entre background e foreground no espaço de cor RGB

Figura 3.3 Poliedro de 128 lados do Primatte

Figura 3.4 Primatte operando como plug-in do Nuke

Figura 3.5 Processo de despill no software Keylight. A imagem

(a) apresenta o vazamento no cabelo do ator e a imagem (b) apresenta o resultado do despill.

Figura 3.6 Matte bruto (a), com erode (b) e com despot (c), no software Keylight.

Figura 3.7 IBK-Color recriando o background (a) a partir da imagem original (b).

Figura 3.8 Retirada do background por subtração, após uso do IBK-Color.

Figura 3.9 Acessórios para o Chromatte: tela tipo cortina (a) e anel de LEDs para a lente da câmera (b).

Figura 4.1 Círculo de amostragem de raio $r$ centrado no pixel 
Figura 4.2 Distribuição das cores como cargas elétricas no espaço de cor P (RGB ou LAB, onde q1, q2, e q3 são cores foreground (com carga elétrica +1), q4 e q5 são cores background, di são distâncias e U é o pixel a ser classificado.

Figura 4.3 Distâncias mínimas de foreground e background no espaço de cor, do ponto $U$ até às superfícies dos clusters, e distância de $U$ até o eixo entre os clusters.

Figura 5.1 Resultados para imagem em alta resolução (a) com o método local baseado em potencial elétrico (c) e o método mais global baseado em clusters (b)

Figura 5.3 Imagem extraída da base de testes de Wang \& Cohen (2007)

Figura 5.4 Imagem extraída da base de testes de Wang \& Cohen (2007) 50

Figura 5.5 Imagem extraída da base de testes de Wang \& Cohen (2007)

Figura 5.6 Imagem extraída da base de testes de Wang \& Cohen (2007)

Figura 5.7 Imagem extraída da base de testes de Wang \& Cohen (2007)

Figura 5.8 Imagem extraída da base de testes de Wang \& Cohen (2007) 
Figura 5.9 Pincel em tempo real desenvolvido para este trabalho, porém sem integração com o sistema chromaless proposto 


\section{Lista de equações}

Equação 2.1 Cor baseado na composição do alpha entre FG e BG. 22

Equação 4.1 Cálculo do raio de busca dinâmica. 43

Equação 4.2 Cálculo do Alpha baseado em potencial elétrico 44

Equação 4.3 Cálculo do potencial de Foreground 44

Equação 4.4 Cálculo do potencial de Background 44

Equação 4.5 Cálculo do alpha por diferença de pontencial 44

Equação 4.6 Cálculo do alpha e confiabilidade baseado na distância 46 
“A Simplicidade é o último degrau da sabedoria"

Khalil Gibran 